\title{
Exploiting DNA Immunization to Generate Polyclonal Antisera to Coronavirus Replicase Proteins
}

\author{
SUSAN C. BAKER, AMORNRAT KANJANAHALUETHAI, NATHAN M. \\ SHERER, DAVID D. AXTELL, AND JENNIFER J. SCHILLER \\ Department of Microbiology and Immunology, Loyola University of Chicago, Stritch School of \\ Medicine, Maywood, IL, USA
}

\section{INTRODUCTION}

Over the last 10 years, a large number of studies have shown that immunization with plasmid DNA encoding an antigen can elicit specific immune responses in animals (reviewed in Donnelly et al 1997; Robinson and Torres 1997). The type of immune response generated depends on several factors, such as the route of inoculation, whether the DNA-encoded antigen remains intracellular or is secreted, and the stability and antigenicity of the protein products. DNA immunization is an attractive alternative to traditional purified protein immunizations because plasmid DNA is relatively easy and inexpensive to generate. However, additional studies are required to determine the optimal route of delivery of plasmid DNA and if additional factors may help "direct" the encoded antigen to elicit a specific type of immune response.

For our studies of the MHV replicase complex, we wanted to exploit DNA vaccine technology to generate polyclonal antisera to replicase products. To this end, we tested 3 different routes of DNA immunization and 2 expression constructs to determine the best conditions to stimulate a strong humoral immune response to our antigen of interest. We found that priming rabbits with plasmid DNA encoding a chimeric cytotoxic $T$ lymphocyte antigen 4-MHV domain 11 fusion protein, termed CTLA4-D11, followed by a booster inoculation with GST-D11 protein elicited a high

The Nidoviruses (Coronaviruses and Arteriviruses).

Edited by Ehud Lavi et al., Kluwer Academic/Plenum Publishers, 2001. 
affinity antibody response and generated polyclonal antisera that recognized MHV replicase proteins by ELISA, Western blot and immunoprecipitation assays.

\section{MATERIALS AND METHODS}

\subsection{Plasmid DNA}

Plasmid pD11 was generated by amplification of the D11 region (MHVJHM nucleotides 9,941-10,204 numbering as per Lee et al 1991 as modified by Bonilla et al 1994) with oligonucleotides containing Not I sites followed by ligation into the pCMV- $\beta$ gal vector (Invitrogen) backbone. Plasmid pCTLA4-D11 was generated by amplification of the rabbit CTLA4 ectodomain (nucleotides 49-525, numbering according to Isono and Seto, 1995) from pCTLA4-Ig (a kind gift from Dr. David Dichek, UCSF, CA). The CTLA4 ectodomain PCR product was digested with Not I and EcoR I and ligated with an EcoR I-Not I D11 fragment and then ligated into the Not I digested pCMV vector. The resultant plasmid was checked for orientation of the insert by digestion with EcoR I and designated pCTLA4-D11.

\subsection{DNA immunization and analysis of antisera}

DNA immunizations were performed essentially as described by Sundaram et al (1996) using an Helios gene gun (BioRad), or by directly injecting $1.0 \mathrm{mg} / \mathrm{ml}$ of plasmid DNA in PBS intramuscularly (IM). The generation of the GST-D11 fusion protein and the resulting anti-D11 sera are described elsewhere (Kanjanahaluethai and Baker 2000). Antibody titer of DNA immunized animals was determined by ELISA to GST-D11 fusion protein and by immunoprecipitation using methods previously described by our laboratory (Schiller et al 1998).

\section{RESULTS AND DISCUSSION}

We wanted to exploit DNA immunization and develop a relatively inexpensive and non-labor intensive method to generate polyclonal antisera in rabbits. Previous studies demonstrated that immunization of rabbits with DNA encoding $\beta$-galactosidase ( $\beta$-gal) did indeed induce specific antibodies that recognized $\beta$-gal protein in an ELISA and Western blot assay (Sundaram et al 1996). In this study, our antigen of interest was the D11 
domain of the MHV-JHM replicase polyprotein. The D11 domain is located just upstream of the MHV 3C-like proteinase (3CLpro) domain. Previously, we showed that 3 CLpro was generated from a p150 precusor polyprotein (Schiller et al 1998). We wanted to generate antibodies specific to the D11 domain to help us determine if p150 extended upstream of the 3CLpro region.

First, we generated pD11 DNA (Figure 1) as described in the Materials and Methods and immunized rabbits using three different routes of inoculation: 1) intramuscular (IM) inoculation of $1 \mathrm{mg}$ plasmid DNA suspended in phosphate buffered saline (PBS) (rabbits 1 and 2);2) IM administration of DNA in PBS with prior adminstration of bupivacaine and co-adminstration of cardiotoxin according to the method of Wells, 1993 (rabbits 3 and 4); and 3) gene gun adminstration of plasmid DNA according to Sundaram et al (1996) (rabbits 5 and 6). Each rabbit was inoculated with DNA at 3 week intervals and blood collected 10 days after each inoculation. As a control, 2 rabbits were injected with purified GST-D11 fusion protein (rabbits 7 and 8, described in detail in Kanjanahaluethai and Baker 2000). Specific antibody response was determined by ELISA to GST-D11 protein. As shown in Table 1, none of the pD11-inoculated rabbits generated a specific antibody response. This was likely because the D11 antigen was not secreted from transfected cells (data not shown). DNA vaccines expressing intracellular proteins are more likely to generate a strong cytotoxic $\mathrm{T}$ lymphocyte response and minimal if any, antibody response.

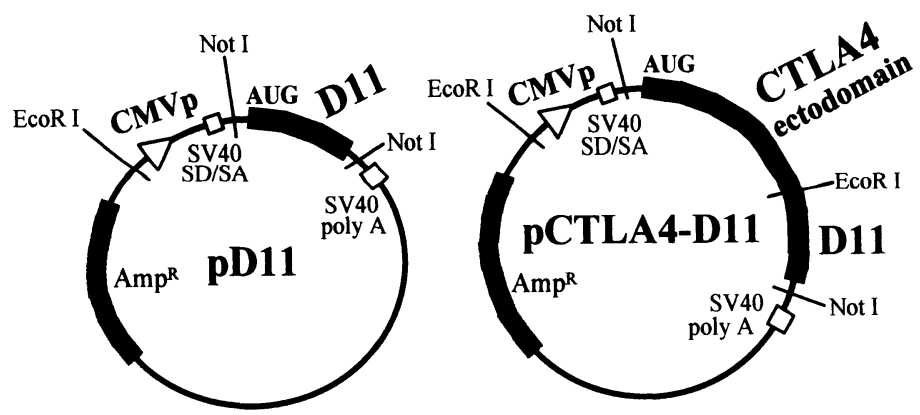

Figure 1. Schematic diagram of the constructs generated for DNA immunization of rabbits. The vector backbone is derived by digestion of pCMV-Bgal (Invitrogen) with Not I and isolation of the vector fragment. The D11 and CTLA4-D11 inserts were derived by PCR amplification as described in the methods section.

To determine if a plasmid DNA encoding a secreted form of D11 would stimulate a humoral immune response in rabbits, we constructed pCTLA4D11 (Figure 1). This plasmid DNA encodes the ectodomain of rabbit CTLA4 in frame with the D11 domain. Studies by Boyle and co-workers showed that DNA vaccines encoding CTLA4-chimeric antigens generated a 
strong humoral immune response, likely because the CTLA4 ectodomain binds to B7 on antigen presenting cells (APCs) (Boyle et al 1998; Linsley et al 1991). Thus, the CTLA4 ectodomain facilitates the secretion of the chimeric antigen and likely directs it to bind APCs.

We tested DNA immunization via IM inoculation (rabbits 9 and 10) and gene gun inoculation (rabbits 11 and 12) for induction of specific antibodies to D11 fusion protein. As seen in Table 1, D11-specific antibodies were induced in all 4 rabbits, with higher titers detected in the gene gun inoculated animals. This is consistent with the results of other investigators who showed that plasmid DNA administered by a gene gun enters dendritic cells, the optimal site for induction of an immune response to the encoded antigen (Torres et al 1997; Akbari et al 1999; Takashima and Morita 1999). Overall, the results of the pD11 and pCTLA4-D11 inoculation studies show that DNA encoding a CTLA4-fusion protein administered via the gene gun stimulates the highest titers of specific antibody (Table 1).

Table 1. Specific antibody response detected in sera isolated from rabbits immunized with GST-D11 protein or with plasmid DNA encoding the MHV-D11 domain.

\begin{tabular}{ccccccc}
\hline & & & \multicolumn{4}{c}{ Titer to GST-D11 protein $^{\mathbf{a}}$} \\
\cline { 4 - 7 } Rabbit & Antigen & Inj/Ad & Pre & s $^{\text {st }}$ & $\mathbf{2}^{\text {nd }}$ & $\mathbf{3}^{\text {rd }}$ \\
\hline \hline 1 & pD11 & IM & $<10$ & n.d. & $<10$ & $<10$ \\
2 & pD11 & IM & $<10$ & n.d. & $<10$ & $<10$ \\
3 & pD11 & IM/bup+CT & $<10$ & n.d. & $<10$ & $<10$ \\
4 & pD11 & IM/bup+CT & $<10$ & n.d. & $<10$ & $<10$ \\
5 & pD11 & gg & $<10$ & $<10$ & $<10$ & $<10$ \\
6 & pD11 & gg & 80 & 80 & 80 & 80 \\
7 & GST-D11 & IM+SC/FA & $<10$ & n.d. & 256,000 & 512,000 \\
8 & GST-D11 & IM+SC/FA & $<10$ & n.d. & 512,000 & $1,024,000$ \\
9 & pCTLA4-D11 & IM & $<10$ & $<10$ & 160 & 320 \\
10 & pCTLA4-D11 & IM & $<10$ & $<10$ & 1,040 & 2,080 \\
11 & pCTLA4-D11 & gg & $<10$ & 140 & 2,080 & 10,240 \\
12 & pCTLA4-D11 & gg & 280 & 1,040 & 10,240 & 40,960 \\
\hline
\end{tabular}

${ }^{\text {a }}$ Preimmune sera (Pre) and sera isolated from blood taken 10-14 days after each of three injections were tested for specific antibody response by ELISA to GST-D11 protein

${ }^{\mathrm{b}}$ Method of injection, IM = intramuscular; bup $+\mathrm{CT}$ = bupivacaine + cardiotoxin; gg = gene gun; $\mathrm{SC}=$ subcutaneous; $\mathrm{FA}=$ Freund's adjuvant

${ }^{c}$ n.d. $=$ not determined

Our next step was to determine if the antibodies generated by DNA immunization were of sufficient affinity to immunoprecipitate viral antigens from MHV-infected cells. HeLa-MHVR cells were infected with MHV-A59 
at a moi of 10 and radiolabeled with $100 \mu \mathrm{Ci} / \mathrm{ml}\left({ }^{35} \mathrm{~S}\right)$-translabel from 3.5$5.5 \mathrm{~h}$ pi. Lysates were prepared and subjected to immunoprecipitation with $5 \mu \mathrm{l}$ of sera from rabbits $1-6$ and $9-12$, and products analyzed by $5-10 \%$ SDS-PAGE. However, no MHV-specific products were detected (data not shown). A review of recent literature revealed that boosting DNA immunized animals with soluble protein stimulates maturation of the antibody response to the specific antigen (Richmond et al 1998; Levtin et al 1997; Boyle et al 1997). We boosted rabbits 2, 4, 9, 10, 11 and 12 with 0.5 mg of purified GST-D11 protein in complete Freund's adjuvant, isolated sera 14 days after the boost and determined the titer to GST-D11 protein by ELISA. Rabbits 2 and 4 had low titer (40-120) consistent with a primary response to immunization (data not shown). In contrast, rabbits 9-12 exhibited 2-10 fold increases in specific antibody titer, consistent with a secondary response to the D11 antigen. We then tested the polyclonal antisera from DNA primed-protein boosted animals to determine if it was of sufficient affinity to immunoprecipitate replicase products from MHVinfected cells as described above. As shown in Figure 2, MHV replicase products $\mathrm{p} 150$ and $>\mathrm{p} 300$ were detected from lysates immunoprecipitated with sera from DNA primed and protein boosted animals (lanes 2 and 4).

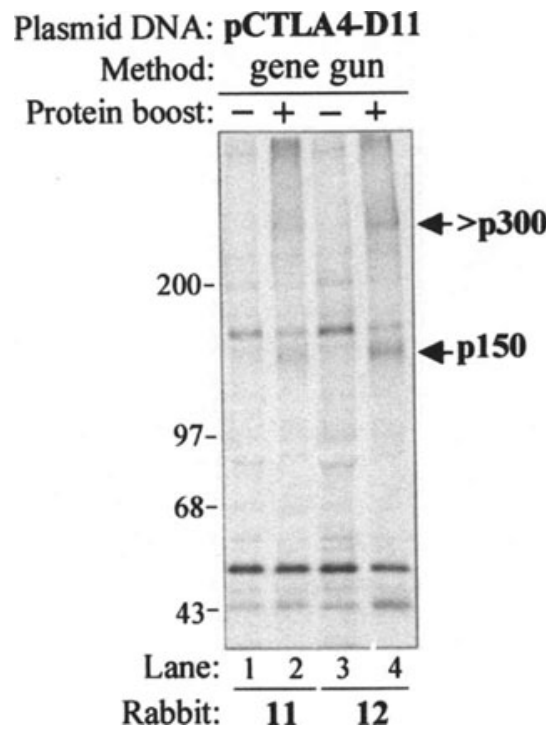

Figure 2. Detection of MHV-specific replicase products with antisera from pCTLA4-D11 primed, GST-D11 protein boosted rabbits. Radiolabeled MHV-infected cell lysates were prepared and subjected to immunoprecipitation as previously described (Schiller et al, 1998). Antisera were from rabbits 11 and 12 after 3 DNA immunizations (-) or after the DNA immunized rabbits had been boosted with $0.5 \mathrm{mg}$ purified GST-D11 protein $(+)$. Products of the immunoprecipitation were analyzed by $5-10 \%$ SDS-PAGE and subjected to autoradiography. MHV-specific products $>$ p300 and p150 are indicated. 
We noted that replicase product p44 was not resolved from background band on this gel. It should also be noted that the pre- and post-protein boosting sera were diluted to identical ELISA titers for this experiment. Thus, immunoprecipitation of the viral replicase product is likely due to affinityboosting of the response, not simply to higher titer of antibody.

Overall, we present data demonstrating the utility of the DNA priming/protein boosting procedure for the rapid generation of high affinity polyclonal antisera in rabbits. This DNA priming/protein boosting approach is likely to facilate the generation of polyclonal antisera to viral or cellular proteins that are challenging to express in sufficient quantity for standard, protein immunization protocols. In addition, this procedure may elicit higher avidity antibodies or antibodies to epitopes that may not be revealed during protein immunization. We anticipate that this approach will help us in our efforts to generate polyclonal antisera to all MHV replicase products and to identify their localization and function in MHV RNA synthesis.

\section{ACKNOWLEDGEMENTS}

We thank John Zaryczny for his assistance with rabbit injection and sera collection. This work was supported by Public Health Service Research Grant AI 32065.

\section{REFERENCES}

Akbari, O., Panjwani, N., Garcia, S., Tascon, R., Lowrie, D., and Stockinger, B. 1999. DNA vaccination: Transfection and activation of dendritic cells as key events for immunity. $J$. Exp. Med. 189:169-177.

Bonilla, P.J., Gorbalenya, A.E., and Weiss, S.R. 1994. Mouse hepatitis virus strain A59 RNA polymerase gene ORF la: Heterogeneity among MHV strains. Virology 198:736-740.

Boyle, J.S., Brady, J.L., and Lew, A.M. 1998. Enhanced responses to a DNA vaccine encoding a fusion antigen that is directed to sites of immune induction. Nature 392:408411.

Boyle, J.S., Silva, A., Brady, J.L., and Lew, A.M. 1997. DNA immunization: Induction of higher avidity antibody and effect of route on T cell cytotoxicity. Proc. Natl. Acad. Sci. USA 94:14626-14631.

Donnelly, J.J., Ulmer, J.B., Shiver, J.W., and Liu, M.A. 1997. DNA vaccines. Annu. Rev. Immunol. 15:617-648.

Isono, T., and Seto, A. 1995. Cloning and sequencing of the rabbit gene encoding T-cell costimulatory molecules. Immunogenetics 42:217-220.

Kanjanahaluethai, A., and Baker, S.C. 2000. Identification of activity of mouse hepatitis virus papain-like proteinase 2. J. Virol. (in press)

Lee, H.-J., Shieh, C.-K., Gorbalenya, A.E., Koonin, E.V., Monica, N.L., Tuler, J., Bagdzhadzhyan, A., and Lai, M.M.C. 1991. The complete sequence (22 kilobases) of 
murine coronavirus gene 1 encoding the putative proteases and RNA polymerase. Virology 180:567-582.

Letvin, N.L., Montefiori, D.C., Yasutomi, Y., Perry, H.C., Davies, M.E., Lekutis, C., Alroy, M., Freed, D.C., Lord, C.I., Handt, L.K., Liu, M.A., and Shiver, J.W. 1997. Potent, protective anti-HIV immune responses generated by bimodal HIV envelope DNA plus protein vaccination. Proc. Natl. Acad. Sci. USA 94:9378-9383.

Linsley, P.S., Brady, W., Urnes, M., Grosmaire, L.S., Damle, N.K., and Ledbetter, J.A. 1991. CTLA-4 is a second receptor for the B cell activation antigen B7. J. Exp. Med. 174: 561569.

Richmond, J.F.L., Lu, S., Santoro, J.C., Weng, J., Hu, S.L., Montefiori, D.C., and Robinson, H.L. 1998. Studies of the neutralizing activity and avidity of anti-human immunodeficiency virus type 1 env antibody elicited by DNA priming and protein boosting. J. Virol. 72:9092-9100.

Robinson, H.L., and Torres, C.A T. 1997. DNA vaccines. Semin. Immunol. 9:271-283.

Schiller, J.J., Kanjanahaluethai, A., and Baker, S.C. 1998. Processing of the coronavirus MHV-JHM polymerase polyprotein: Identification of precursors and proteolytic products spanning 400 kilodaltons of ORFla. Virology 242:288-302.

Sundaram, P., Xiao, W., and Brandsma, J.L. 1996. Particle-mediated delivery of recombinant expression vectors to rabbit skin induces high-titered polyclonal antisera (and circumvents purification of a protein immunogen). Nucl. Acids Res. 24:1375-1377.

Takashima, A., and Morita, A. 1999. Dendritic cells in genetic immunization. J. Leukoc. Biol. 66:350-356.

Torres, C.A.T., Iwasaki, A., Barber, B.H., and Robinson, H.L. 1997. Differential dependence on target site tissue for gene gun and intramuscular DNA immunizations. J. Immunol. 158:4529-4532.

Wells, D.J. 1993. Improved gene transfer by direct plasmid injection associated with regeneration in mouse skeletal muscle. FEBS 332:179-182. 